\title{
RENDA E CONSUMO DE BENS DURÁVEIS NO \\ BRASIL: A APLICAÇÃO DA ESCALA ECD NAS \\ REgIÕES URBANAS DO SUDESTE E NORDESTE, $1996 / 1997^{1}$
}

\author{
Paulo Tafner ${ }^{2}$ \\ Marcelo Costa Ferreira ${ }^{3}$
}

\section{INTRODUÇÃO}

Medir corretamente a renda é extremamente importante, pois a renda, além de ser uma variável que expressa a posição social e econômica de uma família ou indivíduo, é também uma das medidas mais objetivas de bem-estar, sendo usualmente utilizada como variável discriminante para o estabelecimento de políticas sociais com adequada focalização. Mas medir adequadamente a renda individual e/ou familiar em pesquisas amostrais está longe de ser tarefa trivial. Como parece ser consenso entre analistas, unidades familiares mais ricas tendem a sub-declarar a renda - seja por razões culturais ou por temor ao fisco (Hoffmann 1988,51). Este fato foi constatado nos dados do censo demográfico de 1970 (Llunch, 1982) e de 1980 (Hoffmann 1988,51). ${ }^{4}$ Como sabemos, caso o viés - para mais ou para menos - apresen-

1 Os autores gostariam de agradecer a Jose Cláudio Ferreira, Marco Antônio Cavalcanti e Cristiano Fernandes pelas leituras de versões preliminares do presente estudo. Em especial, sem a ajuda de Nelson do Valle Silva este trabalho jamais teria sido feito. Ele forneceu aos autores, de forma muito generosa, o insight acerca da possibilidade do uso do consumo como proxy da renda.

2 Pesquisador da Diretoria de Estudos Macroeconômicos/ IPEA-Rio.

3 Doutorando em Ciência Política na PUC/SP.

4 Existe também a possibilidade das famílias mais pobres declararem uma renda maior do que eles recebem - por questões pessoais, ou por limitações na compreensão do questionário devido à parca escolarização; contudo, este fato não foi identificado nas análises comparativas entre os Censos Demográficos de 1970 e 1980 e outras fontes de dados, como o PIB ou as contas nacionais, segundo Lluch(idem) ou Hoffann (idem) . 
tasse regularidade comportamental, poderíamos fazer os devidos ajustes, obtendo o correto valor da renda. No entanto, não é isso o que ocorre. E se isso é um problema para pesquisadores, torna-se especialmente sensível se o tema é implementação de política públicas voltadas à redução das desigualdades: medidas inexatas de renda podem fornecer recursos a quem deles não precisa ou deixar de levar a quem precisa.

A principal fonte de dados anuais sobre o Brasil é a PNAD/IBGE - Pesquisa Nacional por Amostra Domiciliar. Ela é realizada desde 1967, tendo periodicidade anual desde 1973 - excluindo os anos de censo demográfico. ${ }^{5}$ A PNAD consiste na principal fonte de informação que se dispõe sobre renda no âmbito de pesquisas domiciliares, tendo sido a fonte empírica de diversos estudos sobre as desigualdades no Brasil. Entretanto, diversos estudos acadêmicos de natureza empírica criticam a qualidade das informações sobre renda capturadas pela $\mathrm{PNAD},{ }^{6}$ pois identificam profundas discrepâncias quando as comparam com outras fontes como a POF Pesquisa do Orçamento Familiar -, as Contas Nacionais ou a PPV - Pesquisa de Padrões de Vida. ${ }^{7}$

Barros, Mendonça e Neri (1996, p. 115), por exemplo, constatam a existência de considerável diferença entre a renda familiar nas duas pesquisas (PNAD e POF). Segundo os autores, a maior cobertura de itens de renda no caderno de perguntas ${ }^{8}$ da pesquisa de orçamentos explica a diferença. Também Bonelli e Ramos (1995), concluem pela premente necessidade de se melhorar a qualidade da mensuração da renda tal como apurada pela citada pesquisa.

Pfeffermann e Webb, (1979, p. 15-24), usando dados da PNAD 1972 encontram discrepância na medição da renda pessoal em relação às contas nacionais de $43 \%$, quando se considera a renda monetária. Contudo, esta divergência diminui para $24 \%$, quando a renda não monetária ou de transferências são consideradas, ${ }^{9}$ Segundo eles, a limitação do questionário

5 Há algumas lacunas excepcionais, como no período de execução da ENDEF em 1974/1975 e em 1994, tendo em vista a abrupta redução da taxa de inflação.

6 Todas as pesquisas citadas são ou foram executadas pelo IBGE.

7 IBGE (1981).

8 O caderno de perguntas das POFs costuma ser muito diferente de um questionário de uma pesquisa domiciliar padrão, razão pela qual as duas expressões não são equivalentes.

9 Segundo o mesmo estudo, a inclusão da renda não monetária reduz a discrepância do censo demográfico de 1960 em relação às contas nacionais de $42 \%$ para $31 \%$. 
da PNAD 1972 seria a captação apenas de fatores financeiros, excluindo a renda em espécie. Usando dados da PNAD 1976, a diferença em relação às Contas Nacionais é de $28 \%$, enquanto a ENDEF apresenta discrepância em relação àquela base de $20 \%$. Os autores concluem que ENDEF foi muito mais eficaz para medir renda do que as PNADs de 1973 e 1976; e no caso da PNAD de 1972 para captar rendas não monetárias - apesar de a última ter quesitos para medir todos os tipos de renda. ${ }^{10}$

Figueiredo e Valle Silva (1990) apontam que a mensuração da renda, através dos dados da PNAD, fomenta diversos problemas metodológicos. Medici (1988), mostra a existência de subjetividade das questões relacionadas à renda em algumas versões da pesquisa nos anos 1970. Hoffmann (2000, p. 82) destaca as dificuldades da PNAD em captar a renda em áreas rurais, dada a produção para o autoconsumo - característica que também é salientada por Medici (1988, p. 41), que, não só lamenta a má captação da renda urbana pela PNAD, como a inadequação da mesma, como instrumento para captar rendas rurais. Ainda segundo Hoffmann (idem), a comparação entre o PIB estadual e a renda aferida pela PNAD de 1995, em nível de unidade da federação, o leva a concluir que os estados mais ricos tendem a ter maior subdeclaração de renda.

Rocha (2002), em trabalho exaustivo de análise das PNADs, desde 1967 até 2002, em que avalia as modificações conceituais ocorridas nos quesitos de apuração do rendimento, destaca as limitações na mensuração do rendimento. A autora sugere aumento na quantidade de questões referentes à renda, investigando detalhamento dos rendimentos de origem previdenciária e os rendimentos obtidos de aplicações financeiras. Sugere ainda incrementar a imputação dos dados de rendimentos tanto nos microdados das PNADS, quanto no próprio plano tabular, principalmente no que tange ao auto-consumo e ao não pagamento de moradia - que segundo a autora, consiste num procedimento padrão no cálculo dos agregados nas Contas Nacionais - e nos benefícios complementares ao salário, como ajuda habitacional, tickets de alimentação, vale transporte, educação e saúde.

Dois outros aspectos problemáticos na aferição de rendimentos são destacados por Valle Silva (2003, p. 437): alguns rendimentos, principalmente os relacionados as faixas mais elevadas de renda, são de difícil mensuração e a subdeclaração deliberada de muitos entrevistados pertencentes aos estratos com mais recursos.

10 É importante destacar que até 1976 , as PNAD não tinha cobertura nacional; no caso da PNAD de 1972, os estados da região Norte e Centro-Oeste não foram pesquisados (IBGE, S/D:XX). 
Ferreira, Lanjouw e Neri (2000, p. 10-15) combinando uma série de indicadores da PNAD, POF e PPV para a construção de uma linha de pobreza apontam também algumas limitações dos dados de renda da PNAD. Eles destacam que a PNAD apresenta dificuldades em captar a renda do mercado informal e das áreas rurais. Os autores compararam três indicadores: a) consumo domiciliar per capita; b) renda domiciliar per capita calculada com as variáveis existentes apenas na PPV e PNAD e; c) a mesma variável elaborada com as variáveis existentes no arquivo da PPV compatíveis com as da PNAD e em áreas geográficas comuns de ambas as pesquisas. Apesar de afirmarem que a percepção de submensuração de renda das PNADs é exagerada, destacam que elas tendem a superestimar a pobreza no Brasil e a subestimar a renda, devido ao reduzido número de perguntas no questionário básico sobre aferição de renda.

De fato, as estatísticas apresentadas na tabela 1 revelam que a renda domiciliar mensal ${ }^{11}$ quando medida pela PPV 1996/1997 é pelo menos $50 \%$ maior do que a da PNAD/96 para domicílios em áreas urbanas e eletrificadas das Regiões Sudeste e Nordeste do Brasil. Também a moda da renda da PPV é $167 \%$ maior do que a capturada pela PNAD, corroborando a necessidade de aumentar a precisão da mensuração das estimativas de renda nas PNADs.

Tabela I - ESTATÍSTICAS DESCRITIVAS DA VARIÁVEL RENDA DOMICILIAR NA PNAD E PPV, ÁREAS URBANAS E ELETRIFICADAS DAS REGIÕES SUDESTE E NORDESTE DO BRASIL, 1996-1997*

\begin{tabular}{|c|c|c|c|}
\hline $\begin{array}{l}\text { Pesquisa } \\
\text { Estalisticas } \\
\text { Descritivas }\end{array}$ & $\begin{array}{l}\text { Pesquisa Nacional por } \\
\text { Amosira de 1Jomicilios } \\
\text { (PNAD) - } 1996\end{array}$ & $\begin{array}{l}\text { Pesquisa de Padrôes de Vida } \\
\text { (PI'V). 1996:1997 }\end{array}$ & $\begin{array}{c}\text { Oo de acréscimo da renda da PPV em } \\
\text { relação a } \\
\text { PNAD de } 1996\end{array}$ \\
\hline Média & 1.034 & 1.659 & 60,4 \\
\hline Moda & 112 & 300 & 1679 \\
\hline Iesvio l'tdrâo & 1.568 & 2.709 & 72.8 \\
\hline Mediana & 577 & 887 & 53,7 \\
\hline 1 quartil & 280 & 467 & 66.8 \\
\hline 2 quartil & 577 & 887 & 53.7 \\
\hline 3 quartil & 1.180 & 1.787 & 51,4 \\
\hline$N^{*}$ & 22.938 .511 & $21.532,165$ & \\
\hline Casos Inválidos & 569.764 & 999.637 & \\
\hline
\end{tabular}

FONTE: Pesquisa Nacional por Amostra de Domicílios - 1996 e Pesquisa de Padrões de Vida - 1996/1997. Processamento realizado pelos autores.

* Ponderado pelo peso do domić́lio. Na PNAD/96, a ponderação foi atualizada pela contagem de população de 1996.

11 Conceito de renda domiciliar da PPV 1996/1997: “foram incluídas rendas provenientes dos trabalhos (incluindo rendas extras e benefícios), do auto consumo, renda estimada de aluguel (domicílio próprio ou em aquisição) e de outras fontes" (IBGE 1998). Já na PNAD de 1996: "valor do rendimento em dinheiro e em produtos ou mercadorias, provenientes do trabalho principal, do trabalho secundário e dos demais trabalhos que a pessoa tinha na semana de referência, não sendo investigado o valor da 
Tendo em vista essa submensuração da renda nas PNADs e as dificuldades operacionais de obtenção da "verdadeira" renda do indivíduo e/ou de seu núcleo familiar, algumas alternativas têm sido pensadas, com o intuito de fazer uma checagem indireta - porém aproximada - da renda, a partir da quantificação da posse de bens de consumo duráveis. Esta estratégia poderia ser utilizada nos casos em que a renda não pudesse ser investigada de forma consistente, ou como forma de corrigir a reconhecida submensuração da renda nas PNADS.

A proposta aqui formulada de se obter uma proxy da renda através de medidas indireta não está livre de imperfeições. Uma família pode ter elevada renda sem ter necessariamente muitos bens duráveis. A posse de bens duráveis está associada a fatores culturais, de ciclo de vida da família e até mesmo de fatores climáticos. Outro aspecto que pode limitar a utilização da ECD é a ocorrência de propensão à poupança atipicamente elevada de um indivíduo ou família. Reconhecemos esta limitação, mas como será mostrado nos resultados empíricos, não há indícios de que este fator reduz a qualidade da Escala de Capacidade de Consumo Domiciliar.

Porém, o objetivo deste estudo é propor um instrumento que, afora dimensões conceituais, possa ser utilizado de forma operacional em identificar grupos para o desenho de populações alvo em políticas públicas.

No presente trabalho, cujo objetivo é propor uma escala - a ECD, Escala de Capacidade de Consumo Domiciliar - que estime os rendimentos domiciliares a partir da posse de bens de consumo duráveis, procuramos mostrar através de diversos testes de aderência que essa pode ser uma estratégia adequada para aferição indireta da renda. A parte empírica é feita com dados da Pesquisa de Padrões de Vida (PPV) de 1996/1997.

A PPV é a versão brasileira da pesquisa por amostragem do tipo Living standard measures study - LSMS, ${ }^{12}$ cujo conceito de survey foi idealizado pelo Banco Mundial em 1980, e desde então tem sido realizada em diversos países do mundo. ${ }^{13}$ A PPV, por suas características, oferece um retrato detalhado dos hábitos de consumo da população, além de dispor de conjunto ímpar de informações socioeconômicas. Realizada em 1996/1997 consiste, em termos gerais, numa pesquisa de amostragem de domicílios em dois estágios. No primeiro, foram criados dez estratos geográficos nas regi-

produção para consumo próprio, além da renda de outras fontes, como pensão ou aplicações financeiras" (IBGE, 1997).

12 Grosh e Muñoz (1996) ou Grosh e Glewwe (1996).

13 Costa do Marfim, Rússia, Peru, Vietnã e Tanzânia. Ver a respeito World

Bank, 2002. 
ões Sudeste e Nordeste do Brasil, tendo como base o censo demográfico de 1991. ${ }^{14}$ Depois, foram criados mais três estratos - dentro de cada partição geográfica - a partir da renda média mensal do chefe do domicílio, obtida a partir do questionário da não amostra do Censo Demográfico. Então, os setores foram inseridos em cada estrato. A cada trimestre, foram selecionados de forma aleatória e equiprovável, os domicílios dentro de cada estrato. ${ }^{15}$

$\mathrm{O}$ arquivo de dados utilizado neste estudo engloba apenas os registros relativos aos domicílios pertencentes às áreas urbanas e eletrificadas do Sudeste e Nordeste do Brasil em 1996/1997. Neste caso, foram analisadas informações relativas a 2.296 domicílios das citadas regiões geográficas que continham informações sobre a posse de bens duráveis - dentre as 3.765 unidades domiciliares presentes na população do estudo.

$\mathrm{O}$ presente texto, além dessa introdução, tem mais três seções. $\mathrm{Na}$ próxima seção, discutimos o conceito da ECD - Escala de Capacidade de Consumo Domiciliar - como medida de mensuração indireta da renda. Em seguida, na seção 2 apresentamos e discutimos cinco fortes evidências de que a escala ECD, pode ser utilizada sob certas condições como variável proxy da renda. Na seção final são apresentadas os principais resultados obtidos e as conclusões.

\section{O CONSUMO DE BENS DURÁVEIS E ESCALA DE CONSUMO DOMICILIAR (ECD)}

Esta seção descreve a gênese da Escala de Consumo Domiciliar (ECD), e a sua validade como uma proxy da variável rendimento domiciliar. O princípio da ECD é que existem três padrões de consumo de bens duráveis no Brasil. O primeiro é caracterizado por bens de consumo, com alta disseminação nos domicílios brasileiros - como o fogão. O segundo refere-se a bens mais sofisticados, não tão populares, mas que também não são raros - como máquinas de lavar. $\mathrm{O}$ terceiro consiste em bens caros e de baixa difusão, como microcomputadores ou máquinas de lavar louças. Em síntese, o primeiro padrão agrupa bens de consumo tradicionais, com alta difusão e baixo preço; o segundo refere-se a bens relativamente recentes; e o terceiro é composto por bens caros e de baixa popularização entre os domicílios brasileiros.

14 Caillaux (1996).

15 Albieri, Bianchini e Cardoso (1995). 
Em 1996/1997, os percentuais de posse de bens duráveis nos domicílios investigados pela PPV revelam que um conjunto significativo desses bens tem ampla difusão. São os casos de fogão, geladeira, ventilador e rádio. Contudo, uma enorme gama de outros bens, microcomputadores, aparelhos de ar-condicionado e microondas, ainda são raros nos domicílios brasileiros. Vídeos e máquinas de lavar roupa apresentaram moderada difusão - o que não ocorria com o segundo bem em 1992/1998, conforme (ver tabela 2).

Os domicílios que possuem bens duráveis de maior valor tendem a ter maior renda do que aquelas unidades domiciliares que possuem apenas bens duráveis de maior difusão - e, portanto, mais baratos. Este é o princípio básico da Escala de Capacidade de Consumo Domiciliar (ECD), escala que proponho como proxy da renda domiciliar. ${ }^{16}$

A citada medida embute em seu valor o peso monetário do bem de consumo durável, tendo em vista o seu respectivo ciclo de vida. É de se esperar que um domicílio com máquina de lavar louça, microcomputador, microondas, fogão e geladeira tenha situação socioeconômica mais favorável do que uma unidade domiciliar com fogão, geladeira, vídeo, liqüidificador, TV e rádio. Apesar de o primeiro domicílio ter menos bens, são bens duráveis possuídos mais caros e apresentam menor disseminação na população.

A tabela 2 apresenta os pesos da escala ECD. A coluna central da matriz revela o percentual de unidades domiciliares que têm o bem e a última coluna apresenta os pesos da ECD, que são compostos pela diferença entre $100 \%$ - caso o bem fosse totalmente disseminado - e o percentual efetivo do bem de consumo durável. O peso relativo de cada bem durável é expresso pela diferença percentual entre o máximo possível e a efetiva incidência daquele bem na sociedade. Observe que o peso de cada bem é inversamente proporcional à sua escassez relativa.

A Escala de Capacidade de Consumo Domiciliar (ECD) é dada por:

$$
E C D=\sum_{i=1}^{n}\left(x_{i} p_{i}\right)
$$

$\mathrm{X}=\left\{\begin{array}{l}0, \text { se não tem o bem } \mathrm{i} \\ 1, \text { se tem o bem } \mathrm{i}\end{array} ;\right.$

$\mathrm{P}_{\mathrm{i}}$ é o peso de cada bem de consumo na ECD.

16 Stouffer et al. (1950) e Linn (1989) são bons textos de introdução à elaboração de escalas. 
Como visto, os pesos da ECD são calculados a partir do percentual de disseminação do bem no total dos domicílios investigados. Quanto mais popular um dado bem durável, menor será o seu peso na construção do ECD. Assim, um domicílio que tiver bens menos comuns nos domicílios e portanto com maiores pesos relativos, tenderá a ter maior pontuação na $\mathrm{ECD}$ - e viceversa.

Tabela 2 - RELAÇÃO DOS RESPECTIVOS PESOS DOS BENS DE CONSUMO DURÁVEIS PRESENTES NA ESCALA DE CAPACIDADE DE CONSUMO DOMICILIAR - ECD, BASEADO NA PPV - 1996/1997

\begin{tabular}{|c|c|c|c|}
\hline $\begin{array}{c}\text { Padrões de } \\
\text { Bens de Consumo Duráveis }\end{array}$ & $\begin{array}{l}\text { Bens de consumo } \\
\text { duráveis }\end{array}$ & $\begin{array}{c}\text { \% de Domicílios } \\
\text { que têm o dado } \\
\text { bem }\end{array}$ & $\begin{array}{c}\text { Pesos da ECD } \\
\text { (\% Difusão máxima - \% de } \\
\text { Domicílios que têm o dado bem) }\end{array}$ \\
\hline \multirow{5}{*}{ Grande difusão } & Fogão & 98,9 & 1,1 \\
\hline & TV & 92,7 & 7,3 \\
\hline & Geladeira & 85,7 & 14,3 \\
\hline & Liqüidificador & 82,7 & 17,3 \\
\hline & Rádio & 76,4 & 23,6 \\
\hline \multirow{6}{*}{ Relativa difusão } & Ventilador & 63 & 37,0 \\
\hline & Máquina de Lavar & 39,7 & 60,3 \\
\hline & Roupa & & \\
\hline & Vídeo & 32,7 & 67,3 \\
\hline & Automóvel & 30,7 & 69,3 \\
\hline & Microondas & 15,1 & 84,9 \\
\hline \multirow[b]{5}{*}{ Reduzida difusão } & Aspirador de Pó & 14,7 & 85,3 \\
\hline & Ar Condicionado & 8,7 & 91,3 \\
\hline & Microcomputador & 6,2 & 93,8 \\
\hline & Máquina de Secar & 5,1 & 94,9 \\
\hline & $\begin{array}{l}\text { Máquina de Lavar } \\
\text { Pratos }\end{array}$ & 4,8 & 95,2 \\
\hline Pontuação & ima da ECD & & 842,9 \\
\hline
\end{tabular}

É possível apontar diversas restrições à ECD, já que ela não considera se os bens de consumo foram comprados novos ou não, e em qual quantidade. Além disso, a ECD não leva em conta as marcas de cada bem adquirido pelo domicílio - o que, por vezes é extremamente relevante. No primeiro caso, são poucos os bens de consumo duráveis que não foram comprados novos, segundos os dados da PPV de 1996/1997 - o que é coerente com o estudo de Saboia (1983). Logo, a depreciação dos escores dos bens usados ou adquiridos por doação teria como custo a elaboração de um questionário mais complexo e longo - e um dos requisitos de uma boa mensuração da variável renda consiste na facilidade e rapidez de coleta no questionário junto ao entrevistado. Já no segundo, o questionário da PPV 1996/1997 não inquiriu os entrevistados sobre as marcas dos bens adquiridos. 
Por outro lado, a ECD apresenta uma enorme vantagem sobre os índices ABA ou Abipime. ${ }^{17}$ Ela permite a atualização da escala, a partir do fornecimento de novos escores dos bens de consumo duráveis, como também permite a inclusão de outros bens com um ciclo de vida novo - bens com pouca difusão, mas que estão presentes nos domicílios com maior renda.

Como se sabe, uma boa escala deve ter duas propriedades características: replicabilidade e validade. A primeira se refere à capacidade da escala de mensurar o fenômeno em outras fontes de dados. A segunda é a associação entre a escala e as variáveis que ela pretende representar, Carmines e Zeller (1990). No caso da primeira, uma pesquisa cross section a PPV 1996/ 1997 não pode ser usada como teste para ECD. Entretanto, no caso da segunda, a ECD tem significativa validade, conforme descreve a seção a seguir.

\section{VALIDAÇÃO DA ECD}

São apresentadas cinco evidências que podem corroborar a qualidade da escala ECD como proxy da variável renda domiciliar. A primeira e mais simples consiste na similaridade dos histogramas das distribuições de renda domiciliar e da ECD captados na PPV de 1996/1997, como mostra o gráfico 1. Concentração à esquerda da distribuição revelando ocorrência de muitos domicílios com bens de baixo valor (ou renda baixa), e poucas unidades domiciliares com bens duráveis de grande valor (ou rendas mais elevadas).

\section{Gráfico 1 - HISTOGRAMAS DAS DISTRIBUIÇÕES DA RENDA* E DA ECD NOS DOMICÍLIOS DAS ÁREAS URBANAS E ELETRIFICADAS DAS REGIÕES SUDESTE E NORDESTE DO BRASIL, 1996-1997}

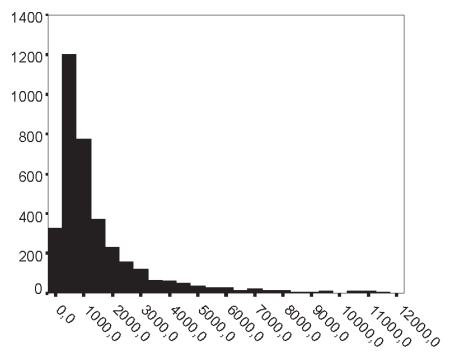

Renda domiciliar

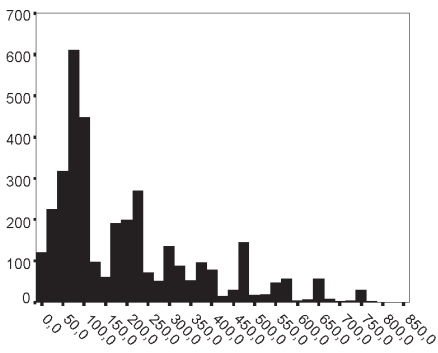

Escala ECD

FONTE: Pesquisa de Padrões de Vida - IBGE

* Exclui domicílios com rendas maiores do que R\$12.000.

Processamento realizado pelo autor.

17 Devo destacar, contudo, que o existe uma diferença crucial entre o conceito da ECD em relação as escalas $\mathrm{ABA} / \mathrm{Abipime:} \mathrm{enquanto} \mathrm{a} \mathrm{primeira} \mathrm{tem} \mathrm{como}$ 
A segunda consiste na comparação dentre diversas estatísticas descritivas. Como se pode observar na tabela 3 são muito semelhantes as estatísticas de renda e da ECD. As diferenças entre as proporções nos $2^{\circ} / 1^{\circ}$, $3^{\circ} / 2^{\circ}, 4^{\circ} / 3^{\circ}$ e $5^{\circ} / 4^{\circ}$ quintis da renda domiciliar com os respectivos quintis da ECD são zero - uma evidência que atesta a qualidade da ECD como proxy da renda domiciliar.

Tabela 3 - QUADRO COMPARATIVO DAS ESTATÍSTICAS DESCRITIVAS DA RENDA DOMICILIAR E DA ECD

\begin{tabular}{c|c|c|c|c|c|c}
\hline & \multicolumn{2}{|c|}{ Estatísticas Descritivas } & & \multicolumn{2}{|c|}{ Razôs } & \\
\hline & Renda & ECD & & Renda & ECD & Renda - ECD \\
\hline Média & 1659,10 & 195,90 & mediámediana & 1,87 & 1,47 & 0,40 \\
\hline Mediana & 887,60 & 132,90 & Mediana Desvio Padrão & 0,33 & 0,77 & $-0,44$ \\
\hline Desvio Padrão & 2721,7 & 173,3 & & & & \\
\hline C. V. & 164,0 & 88,5 & & & & \\
\hline 20 & 406,20 & 63,60 & 20,40 & 0.58 & 0.63 & $-0,05$ \\
\hline 40 & 698,70 & 100,60 & 40,60 & 0,62 & 0,53 & 0,09 \\
\hline 60 & 1126,80 & 191,20 & 6080 & 0,52 & 0,61 & $-0,09$ \\
\hline 80 & 2155,10 & 313,50 & & & & \\
\hline
\end{tabular}

A terceira evidência decorre do ajuste da ECD aos princípios de um escalograma de Guttman. De acordo com a teoria da elaboração das escalas de mensuração, Guttman (1974, p. 144), uma boa escala deve ter uma ordenação dos atributos; além disso, uma das qualidades de uma boa escala deve ser a unidimensionalidade da mesma. Em outras palavras: a escala deve apenas captar o fenômeno que ela está mensurando.

A comprovação da unidimensionalidade de uma escala que siga os procedimentos de um escalograma de Guttman ocorre pela associação entre os itens das variáveis que a escala mensura com os atributos da própria escala. Segundo Benzecri et alli (1976, p. 195; 250-251; 338; 483; 485), a unidimensionalidade de uma escala é identificada pela associação presente num diagrama de análise de correspondência ${ }^{18}$ entre os itens que ela mensura e a própria escala, numa relação semelhante a uma parábola.

É o que ocorre nos Gráficos 5 e 6. Eles mostram o cruzamento entre a ECD padronizada e a propriedade dos bens de consumo. Neste caso, o primeiro gráfico citado mostra a ECD padronizada em três categorias representativas do $1^{\circ}, 2^{\circ}$ e $3^{\circ}$ tercis da ECD; no segundo, em cinco atributos - correspondentes aos $1^{\circ}, 2^{\circ}, 3^{\circ}, 4^{\circ}$ e $5^{\circ}$ quintis da $\mathrm{ECD}$, respectivamente.

objetivo mensurar renda, a segunda pretende medir a posição socioeconômica, tendo em vista a propensão ao consumo. Maiores informações sobre as escalas ABA ou Abipime podem ser encontradas em Jannuzzi e Baeninger (1996) ou Mattar (1995).

${ }^{18} \mathrm{O}$ diagramas de análise de correspondência aqui apresentados foram realizados no SPSS para Windows, versão 9.0. 
Em ambos os diagramas de análise múltipla de correspondência, a associação entre a ECD e os itens que ela pretende mensurar reside numa parábola.

Gráfico 2 - ANÁLISE MÚLTIPLA DE CORRESPONDÊNCIA- ECD PADRONIZADA E POSSE OU NÃO DE BENS DE CONSUMO DURÁVEIS

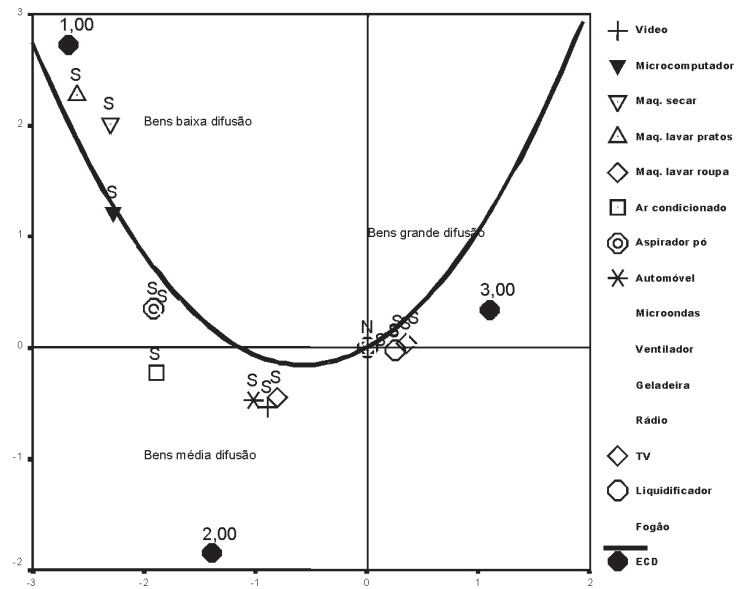

Legenda: $1: 5$ quintil da ECD; 2: 4 quintil da ECD; $3: 3$ quintil da ECD; 4: 2 quintil da ECD e 5: 1 quintil da ECD

Gráfico 3 - ANÁLISE MÚLTIPLA DE CORRESPONDÊNCIA-ECD PADRONIZADA E POSSE OU NÃO DE BENS DE CONSUMO DURÁVEIS

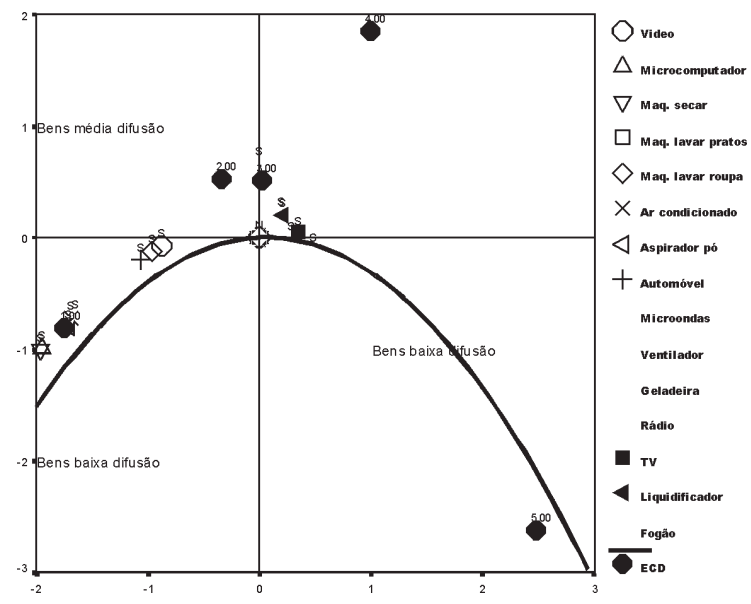

Legenda: 1: 5 quintil da ECD; 2: 4 quintil da ECD; $3: 3$ quintil da ECD; 4: 2 quintil da ECD e 5: 1 quintil da ECD 
A quarta evidência é a comparação entre dois modelos econométricos de mínimos quadrados ordinários (MQO).$^{19} \mathrm{O}$ primeiro tem como variável dependente a ECD; enquanto o segundo, a renda domiciliar. Em ambos, as variáveis independentes são as características do chefe do domicílio e de sua respectiva unidade domiciliar. A qualidade da ECD seria atestada pela semelhança entre a magnitude das estimativas e equivalência entre os sinais dos coeficientes das variáveis explanatórias.

As variáveis independentes foram escolhidas tendo em vista os principais determinantes da renda descritos pela literatura: ciclo de vida da família, mensurado pela idade do chefe: Derrick e Lehfeld, (1980) ou Varady (1990), Lawson (1991); gênero: Ramos e Soares (1994) ou Ramos (1994); cor: Valle Silva (1980 ou 1985) e Hasenbalg et alli (1999, p. 184-216); escolaridade: (GEHZ E BECKER, 1975), Soares (2001, p. 13), Ferreira e Barros (2000), Barros, Corseuil e Mendonça (1999), (BONELLI E RAMOS, 1995, p. 369), Barros e Ramos (1992) ou Langoni (1973); ocupação: Barros, Machado e Mendonça (1997), Ramos (1993, p.61-76) ou (SOUZA, 1980, p.391); e tamanho da familia: (SOUZA, 1980, p. 391).

A composição desses diversos fatores indicaria que chefes do gênero feminino teriam menores chances de obter maior renda em comparação com os chefes masculinos; a idade do chefe - considerada como proxy do tempo no ciclo de vida de uma família - apresenta relação positiva com os renda, dado que chefes na idade dos 30 aos 40 anos teriam mais chances de obter mais renda do que chefes novos ou anciãos; chefes brancos, maiores chances do que os não brancos; chefes mais escolarizados, mais do que os menos escolarizados; e o mesmo nas profissões de maior status sócioocupacional, em relação àquelas de baixo status; domicílios com expressiva proporção de moradores por cômodo teriam menores chances de alcançar maiores rendas. A tabela 4 apresenta as variáveis, com seus respectivos formatos, e estatísticas descritivas; já a tabela 5 descreve os dois modelos econométricos aqui analisados.

19 Os modelos econométricos aqui apresentados foram calculados no SAS. Os cálculos das modelagens efetuados neste estudo foram ponderados pela fração amostral do peso do domicílio. 
Tabela 4 - DESCRIÇÃO E FORMATO DAS VARIÁVEIS INDEPENDENTES²0

\begin{tabular}{|c|c|c|c|c|c|c|c|}
\hline Descrição & Formato & média & Mediana & $\begin{array}{l}\text { desvio } \\
\text { padrão }\end{array}$ & Mínimo & máximo & Obs \\
\hline \multicolumn{8}{|l|}{ Características do Chefe } \\
\hline Idade & Númerica & 46.2 & 44 & 15,7 & 15 & 95 & 3765 \\
\hline Sexo (Feminino) & \begin{tabular}{|l|} 
Dummy \\
\end{tabular} & 0,24 & \multicolumn{4}{|l|}{ 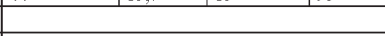 } & 3765 \\
\hline Cor (Branco) & Intervalar & 0,61 & & 3209 \\
\hline Anos de Estudo & Numérica & 6,7 & \multicolumn{4}{|c|}{4,0} & 3301 \\
\hline Ocupação & Dummy & & \multirow{8}{*}{\multicolumn{4}{|c|}{ Não se aplica }} & 3765 \\
\hline Profissões Universitárias & Dummy & 0,03 & & & & & 3765 \\
\hline Dirigentes & Dummy & 0,08 & & & & & 3765 \\
\hline Empresários Empregadores & Dummy & 0,1 & & & & & 3765 \\
\hline Empresários por Conta Própria & Dummy & 0,04 & & & & & 3765 \\
\hline Téenicos Artistas e Supervisão Manual & Dummy & 0,2 & & & & & 3765 \\
\hline Não Mar 2 al de Rotina & Dummy & 0,3 & & & & & 3765 \\
\hline Manuais $^{1}$ & Dummy & 0,4 & & & & & 3765 \\
\hline \multicolumn{8}{|l|}{ Características do Domicilio } \\
\hline Pessoas $/ \mathrm{N}^{0}$ de Cômodos ${ }^{22}$ no Domicílio & Numérica & 1,3 & 1 & 0,9 & 0,11 & 10 & 3764 \\
\hline
\end{tabular}

A tabela 5 mostra os resultados dos modelos econométricos. As estimativas são apresentadas sobre os erros-padrão que estão entre parênteses. Tanto a ECD quanto a renda domiciliar tendem a ser menores nos domicílios com chefia feminina, com elevada proporção de pessoas por cômodo e nas ocupações de menor prestígio socioeconômico. Já as famílias com chefe de cor branca, escolarizado e exercendo profissões de maior status, tendem a ter maiores renda ou pontuação na ECD. As estimativas dos modelos analisados são coerentes com o previsto pela literatura.

20 Proprietários rurais foi a categoria de referência para a elaboração das variáveis ocupacionais binárias.

${ }^{21}$ Esta categoria inclui as seguintes ocupações:manual em indústrias modernas, manual em indústrias tradicionais, manual no terciário, ambulantes, serviços domésticos e proprietários rurais.

banheiros.

22 Inclui sala e quartos do domicílio, mas não contabiliza cozinhas e 
TAFNER, P; FERREIRA, M. C. Renda e consumo de bens duráveis no Brasil...

Tabela 5 - ESTIMATIVAS DOS MODELOS DE REGRESSÃO LINEAR MÍNIMOS QUADRADOS ORDINÁRIOS VARIÁVEIS DEPENDENTES: ECD E RENDA DOMICILIAR EM DOMICÍLIOS COM ENERGIA ELÉTRICA EM ÁREAS URBANAS DO NORDESTE E SUDESTE DO BRASIL 1996/ 1997

\begin{tabular}{|c|c|c|c|c|c|c|}
\hline & \multicolumn{6}{|c|}{ Modelos } \\
\hline \multirow[t]{2}{*}{ Variáveis } & \multicolumn{2}{|l|}{ I } & \multicolumn{2}{|l|}{ II } & \multicolumn{2}{|l|}{ III } \\
\hline & FCI & $\begin{array}{l}\text { Renda } \\
\text { Domiciliar }\end{array}$ & $\mathrm{FCl}$ & $\begin{array}{l}\text { Renda } \\
\text { Domicili } \\
\text { ar }\end{array}$ & $\mathrm{F}(\mathrm{CI})$ & $\begin{array}{l}\text { Renda } \\
\text { Domicilias }\end{array}$ \\
\hline \multicolumn{7}{|l|}{ Caracteristicas do Chefe do Domicilio } \\
\hline Idade & $\begin{array}{l}0.61 \\
(0,18) \\
\end{array}$ & $\begin{array}{l}8.2 \\
(2.8)\end{array}$ & $\begin{array}{l}1.1 \\
(0.17)\end{array}$ & $\begin{array}{l}20.2 \\
(3.0)\end{array}$ & $\begin{array}{l}2.4 \\
(0.18) \\
\end{array}$ & $\begin{array}{l}36.6 \\
(3.4)\end{array}$ \\
\hline Sexo (teminino) & & & $\begin{array}{l}-32.6 \\
(5.8)\end{array}$ & $\begin{array}{l}-418.0 \\
(101.0)\end{array}$ & $\begin{array}{l}-42.7 \\
(5.9)\end{array}$ & $\begin{array}{l}-484.2 \\
(108.2)\end{array}$ \\
\hline Cor (Branco) & & & $\begin{array}{l}83.9 \\
(5.0)\end{array}$ & $\begin{array}{l}412,4 \\
(87,7)\end{array}$ & $\begin{array}{l}57.6 \\
(5.0)\end{array}$ & $\begin{array}{l}99,2^{\mathrm{fls}} \\
(93.13)\end{array}$ \\
\hline Anos de Estudo & & & & & $\begin{array}{l}17.6 \\
(0.7)\end{array}$ & $\begin{array}{l}214.8 \\
(12.8) \\
\end{array}$ \\
\hline \multicolumn{7}{|l|}{ ()upaçâo } \\
\hline Prolissões Universitárias & & & $\begin{array}{l}257.6 \\
(14.0)\end{array}$ & $\begin{array}{l}3878,3 \\
(240.0)\end{array}$ & $\begin{array}{l}70.3 \\
(14.5)\end{array}$ & $\begin{array}{c}1721,1 \\
(263.3)\end{array}$ \\
\hline Dirigentes & & & $\begin{array}{l}92.4 \\
(6.2)\end{array}$ & $\begin{array}{l}1497.76 \\
(107.2)\end{array}$ & $\begin{array}{l}36.7 \\
(6.0)\end{array}$ & $\begin{array}{l}843.9 \\
(110.5)\end{array}$ \\
\hline Empresários Fimpregadores & & & $\begin{array}{l}80.5 \\
(4.6)\end{array}$ & $\begin{array}{l}1170.1 \\
(79,1)\end{array}$ & $\begin{array}{l}47.7 \\
(4.4)\end{array}$ & $\begin{array}{l}790.0 \\
(80.1)\end{array}$ \\
\hline Fimpresários por Con1a Própria & & & $\begin{array}{l}69.5 \\
(12.2)\end{array}$ & $\begin{array}{l}3842^{155} \\
(211.4)\end{array}$ & $\begin{array}{l}31.8^{\text {p:0.05 }} \\
(119)\end{array}$ & $\begin{array}{l}121.7^{15} \\
(216.0)\end{array}$ \\
\hline Técnicos Artistas e Supervisão Manual & & & $\begin{array}{l}35.4 \\
(3.0)\end{array}$ & $\begin{array}{l}343.8 \\
(51.8)\end{array}$ & $\begin{array}{l}9.2^{1000} \\
(2.9)\end{array}$ & $\begin{array}{l}67.7^{\text {IL }} \\
(53.4)\end{array}$ \\
\hline Não Manual de Rotina & & & $\begin{array}{l}9.1 \\
(1.8)\end{array}$ & $\begin{array}{l}124.5 \\
(32.9)\end{array}$ & $\begin{array}{l}-2.6^{\mathrm{rls}} \\
(1.7)\end{array}$ & $\begin{array}{l}4,2^{1 L i} \\
(33.1)\end{array}$ \\
\hline Manuais & & & $\begin{array}{l}4.7^{15} \\
(6.5)\end{array}$ & $\begin{array}{l}195,1^{115} \\
(114.7)\end{array}$ & $\begin{array}{l}-8.0^{1.5} \\
(6.4)\end{array}$ & $\begin{array}{l}157.1^{115} \\
(120.8)\end{array}$ \\
\hline \multicolumn{7}{|l|}{ Características do Domicílio } \\
\hline 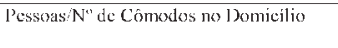 & & & & & $\begin{array}{l}-16.9 \\
(28)\end{array}$ & $\begin{array}{l}-20.6^{1 \mathrm{si}} \\
(54,4)\end{array}$ \\
\hline Constante & $\begin{array}{l}168.5 \\
(8.7) \\
\end{array}$ & $\begin{array}{l}1287.1 \\
(141,6)\end{array}$ & $\begin{array}{l}58.7 \\
(11.2)\end{array}$ & $\begin{array}{l}-27.0^{\mathrm{ns}} \\
(197.4)\end{array}$ & $\begin{array}{l}-28,0^{100.05} \\
(14,3)\end{array}$ & $\begin{array}{l}-1554.2 \\
(2601,1)\end{array}$ \\
\hline $\mathrm{R}^{2}$ ainstacio & 0.03 & 0,02 & 0.28 & 0.18 & 0.42 & 0.23 \\
\hline $\mathrm{F}$ & 11.7 & 8.0 & 146.9 & 76.2 & 1807 & 81.5 \\
\hline$N$ & 3746 & 3549 & 3746 & 3545 & 3286 & 3104 \\
\hline
\end{tabular}

A quinta e última evidência reside na análise do diagrama da renda domiciliar e a ECD - ambas na escala log de base neperiana, gráfico 5. O diagrama de dispersão revela que a existência de uma relação linear entre o Ln da ECD e o Ln da renda domiciliar. 


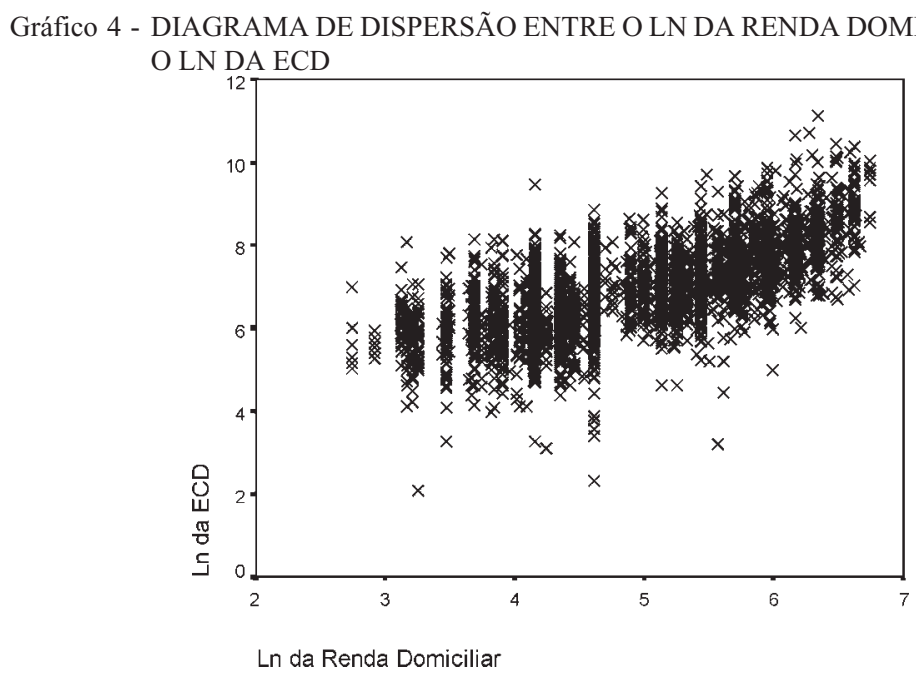

Contudo, existem casos com alta renda domiciliar, mas com baixa ECD - 20 casos; e vice-versa - 114 casos que não foram apresentados no gráfico acima, no qual mais da metade das informações sobre escolaridade e ocupação do chefe está ausente. No primeiro caso, no qual o Ln da renda é menor do que 2,5 , estão domicílios que são, na sua maioria, chefiados por trabalhadores manuais de indústrias tradicionais ou do setor de serviços, além de empregados rurais. No segundo, os casos desviantes consistem nas seguintes ocupações: técnicos artistas e supervisão manual, manual em indústrias tradicionais, manual no setor terciário e trabalhadores rurais que têm alta renda, e baixo consumo de bens duráveis.

Um modelo de regressão linear simples entre o Ln da ECD, variável dependente, e do Ln da renda, independente, apresenta um $\mathrm{R}^{2}$ de 0,42. Mesmo com a exclusão de casos desviantes, e com a inserção de uma variável independente idade binária (onde a idade do chefe com mais de 40 anos= 1), o $\mathrm{R}^{2}$ resultante não passou de 0,49 . A análise deste fato revela que, não obstante as evidências da qualidade da ECD como proxy da renda, a associação entre consumo e renda como estratégia de mensuração de renda requer um estudo mais detalhado, do qual a escala que propomos seria apenas um primeiro passo. Entretanto, a correlação linear entre o Ln da ECD com o Ln da renda per capita é de 0,68 . 


\section{CONCLUSÃO}

O presente artigo teve como objetivo propor uma escala proxy da variável renda domiciliar. A relevância desta estratégia de inquérito da renda ocorre porque famílias e/ou indivíduos com grandes rendas tendem a subdeclarar os valores que recebem. Estimativas equivocadas da renda tendem a enviesar o foco das políticas públicas de desigualdades sociais, além de reduzir a qualidade dos estudos socioeconômicos que tenham como variável de análise a renda.

Diversos estudos da literatura sobre distribuição de renda apontam as distorções da renda da PNAD, quando comparados com outras pesquisas, como a ENDEF, POFs, Contas Nacionais. No caso desta pesquisa, as estimativas da PPV, quando comparadas com as da PNAD, revelam que esta última pesquisa tende a subestimar rendas domiciliares.

A escala aqui proposta, bastante simples em termos de implementação e utilização, consiste do somatório de pesos da difusão de bens de consumo duráveis em domicílios, peso este que é calculado a partir da diferença entre $100 \%$ - difusão máxima que um bem pode obter - e o percentual de unidades domiciliares que têm um dado bem. Domicílios com muita renda tendem a ter bens de consumo mais caros e que, portanto, apresentam difusão menor nas unidades familiares brasileiras.

Cinco evidências apontam a qualidade da ECD como variável proxy da renda familiar: 1) semelhança entre as distribuições de renda da ECD e da renda domiciliar; 2) estatísticas descritivas idênticas, entre a ECD e a renda domiciliar; 3) a associação entre a posse de bens de consumo duráveis e a ECD apresenta o formato de uma parábola num diagrama de análise múltipla de correspondência, o que assegura a unidimensionalidade da ECD, ou seja, a ECD mensura apenas e somente a posse de bens duráveis; 4) Os modelos econométricos que têm como clássicas variáveis independentes na análise dos determinantes da renda, como idade, cor, escolaridade, ocupação, número de cômodos, entre outras; como a ECD e renda domiciliar são equivalentes; e 5) a análise de uma regressão linear simples entre a renda domiciliar, variável dependente, e a ECD, variável independente.

Acreditamos que o debate envolvendo alternativas e aprimoramentos metodológicos para a melhoria da qualidade de medição da renda é fundamental não apenas do ponto de vista acadêmico, mas de seus impactos no estabelecimento de políticas públicas. Um exemplo disto é que não só a utilização da ECD pode ser um mecanismo de correção de rendas subdimensionadas, como também as variáveis da escolaridade do chefe do 
domicílio e o número de cômodos poderiam ser usados como parâmetros para políticas de redução das desigualdades sociais, em combinação com informações oriundas da análise de futuras amostras de uso público do Censo Demográfico brasileiro que tenham um alto grau de detalhamento geográfico, algo semelhante à amostra de uso público de $3 \%$ do Censo demográfico de 1980 - IBGE (1985).

\title{
RESUMO
}

O objetivo deste estudo é propor uma escala que aumente a qualidade da mensuração da variável renda em pesquisas domiciliares: a ECD, (Escala de Capacidade de Consumo Domiciliar). Ela atribui pesos, de acordo com a difusão de bens duráveis em um dado domicílio, considerando o ciclo de vida de um bem durável. A qualidade desse indicador foi verificada a partir de algumas evidências que são apresentadas e discutidas no texto. A análise empírica utiliza os dados da Pesquisa de Padrões de Vida (PPV), 1996/1997 - IBGE. Conclui-se pela pertinência do uso da escala ECD como uma estratégia complementar de mensuração da renda domiciliar, sobretudo em casos em que a captura da informação apresenta dificuldades estruturais.

Palavras-chave: renda, desigualdades sociais, padrões de vida, consumo, indicadores e escalas.

\begin{abstract}
The purpose of this paper is to present a Consumer household capacity scale (ECD) which may improve the quality of earnings measure in Brazilian national household survey, PNAD. The scale is weighted according the presence or not of durable consumer goods. To show how useful this approach can be some evidences of the adherence were presented and discussed. We conclude that ECD Scale is very useful as an auxiliary strategy to get indirect earnings measures. The adequacy of ECD's strategy is specially important when one faced with structural difficulties to capture household earnings.

Key-words: earnings, inequality, standard patterns, consumer, social indicators and scales.
\end{abstract}




\section{REFERÊNCIAS}

ALBIERI, S., BIANCHINI, Z. M.; CARDOSO, R. L. Pesquisa sobre padrões de vida: planejamento da amostra. Rio de Janeiro: Departamento de Metodologia, IBGE, 1995. Mimeografado.

BARROS, R. P.; CORSEUIL, C. H.; MENDONÇA, R. Uma análise da estrutura salarial brasileira baseada na PPV. Rio de Janeiro: Ipea, dez. 1999. Texto para discussão n. 689.

; MACHADO, A. F.; MENDONÇA, R. A desigualdade da pobreza: estratégias ocupacionais e diferenciais por gênero. Rio de Janeiro: Ipea, jan. 1997. Texto para discussão n. 453.

.; MENDONÇA, R.; NERI, M. An evaluation of the measurement of income and expenditure household surveys: POF versus PNAD. In: ENCONTRO BRASILEIRO DE ECONOMETRIA. SOCIEDADE BRASILEIRA DE ECONOMETRIA, 17., 1996. Anais... p. 105-129.

.; RAMOS, L. A note on the temporal evolution of the relationship between wages and education among Brazilian prime-age males: 1976-1989. In: ENCONTRO NACIONAL DE ECONOMIA, 20, 1992, Campos do Jordão: Anpec,. p. 49-62.

BENZÉCRI, J. P. et al. L'analyse des donnés: leçons sur l'ánalyse factorielle et la reconnnaissance des formes et travaux du laboratoire de statistique de 1'Université de Paris VI. Paris: Dunod, 1976.

BONELLI, R.; RAMOS, L. Distribuição de renda no Brasil: avaliação das tendências de longo prazo e mudanças na desigualdade desde meados dos anos 1970. Revista Brasileira de Economia, Rio de Janeiro: FGV-RJ, v. 49, n. 2, p. 353-373, 1995.

CAILlAUX, E. L. Pesquisa sobre padrões de vida: 1996-1997. In: CONFEST. Textos para Discussão: As inovações nas informações sociais e econômicas, v. 2, Rio de Janeiro, 1996. Mimeografado.

CARMINES, E. G.; ZELLER, R. A. Reliability and validity assessment. California: Sage, 1990.

DERRICK, F. W.; LEHFELD, A. The family life cycle: an alternative approach. Journal of Consumer Research, v. 7, p. 214-217, Sept. 1980.

FERREIRA, F. H. G.; LANJOUW, P.; NERI, M. A new poverty profile for Brazil using PPV, PNAD and Census data. Rio de Janeiro: Departamento de Economia; PUC-Rio, mar. 2000. Texto para discussão n. 418.

FIGUEIREDO, J. B.; VALLE SILVA, N. População, família e renda no Rio de Janeiro (1970/1985). Revista Brasileira de Economia. Rio de Janeiro: FGV-RJ, v. 44, n. 1, p. 85106, 1990.

FERREIRA, F. H. G.; BARROS, R. P. La educación y la distribución del ingreso en el Brasil urbano, 1976,1996. Revista de la Cepal, n. 71, ago. 2000.

GHEZ, G. R.; BECKER, G. S. The allocation of time and goods over life cycle. National Bureau of Economic Research, New York: Columbia University Press, 1975.

GROSH, M. What use are they? Multitopic household survey data in policymaking. Draft. [S.1.:s.n.], [19-]. 
.; MUÑOZ, J. A manual for planning and implementing the living standards measurement study surveys. LSMS Working Paper, n. 126, The World Bank, 1996.

.; GLEWWE, P. A guide to living standard measurement study surveys and their data sets. Série Seminários, n. 1, 1996. Diretoria de Pesquisa, IPEA.

GUJARATI, D. N. Basic econometrics. 3. ed. New Aster: McGraw-Hill, 1995.

GUTTMAN, L. L. The basis for scalogram analysis. In: MARANELL, G. M. Scaling: a sourcebook for behavioral scientists. Chicago: Aldine, 1974. p.142-171.

HASENBALG, C.; VALLE SILVA, N.; LIMA, M. Cor e estratificação social. Rio de Janeiro: Contracapa, 1999.

HOFFMANN, R. Mensuração da desigualdade e pobreza no Brasil. In: HENRIQUES, R. Desigualdade e pobreza no Brasil. Rio de Janeiro: Ipea, 2000. p. 81-107.

Desigualdade entre estados na distribuição da renda no Brasil. Economia Aplicada, v. 1, n. 2, p. 281-296, 1997.

. A subdeclaração de renda. São Paulo em Perspectiva, São Paulo: Seade, v. 2, n. 1, p. 50-54, 1988.

IBGE. CD-Rom dos microdados da PPV-1996-1997. Rio de Janeiro: IBGE. 1998.

CD-Rom dos microdados da PNAD de 1996. Rio de Janeiro: IBGE. 1997.

Amostra de uso público do Censo Demográfico de 1980: metodologia e manual do usuário. Rio de Janeiro: IBGE, 1985.

. Metodologia da pesquisa nacional por amostragem de domicílios na década de 70. Rio de Janeiro: IBGE, 1981.

JANNUZZI, P. M; BAENINGER. Qualificação socioeconômica e demográfica das classes da escala Abipime. Revista de Administração, São Paulo, v. 31, n. 3, p. 82-90, 1996.

LANGONI, C. Distribuição de renda e desenvolvimento econômico do Brasil. Rio de Janeiro: Expressão e Cultura, 1973.

LAWSON, R. Patterns of tourist expenditure and types of vacations across the family life cycle. Journal of Travel Research, p. 12-18, Spring, 1991.

LINN, R. Educational measurement. American Council on Education. [S.1.]: Macmillan, 1989.

LLUNCH, C. Sobre medições de renda a partir dos censos e das contas nacionais no Brasil. Pesquisa e Planejamento Econômico, Rio de Janeiro, v. 12, n. 1, p. 133-148, abr. 1982.

MATTAR, F. N. Análise crítica dos estudos de estratificação sócio-econômica de ABAAbipime. Revista de Administração, São Paulo, v. 30, n. 1, p. 57-74, 1995.

MEDICI, A. C. A mensuração da subjetividade: notas sobre a variável renda nas PNADs. In: SAWYER, D. PNADs em foco. [S.1.]: Abep, 1988. p.121-145.

PFEFFERMANN, G. P.; WEBB, R. The distribution of income in Brazil. Working paper, n. 356, Sept. 1979.

RAMOS, L.; SOARES, A. L. Participação da mulher na força de trabalho e pobreza no Brasil. Ipea, 1994. Textos de discussão n. 350.

RAMOS, L. Poverty in Brazil in the 80s. Ipea, 1994. Textos de discussão n. 361. 
. A distribuição de renda no Brasil: 1976/1985. Rio de Janeiro: Ipea, 1993.

ROCHA, S. A investigação do rendimento na PNAD - comentários e sugestões à pesquisa nos anos 2000. Rio de Janeiro: Ipea, ago. 2002. Texto de discussão n. 899.

SABOIA, J. L. M. As causas da difusão da posse dos bens de consumo duráveis no Brasil. Rio de Janeiro: Ipea; PNPE, 1983.

SOARES, S. S. D. Simulando - o mundo maravilhoso das distribuições contrafactuais. Brasília: Ipea, fev. 2001. Texto para discussão n. 780.

SOUZA, A. M. Despesas familiares em educação: um estudo empírico. Revista Brasileira de Economia, Rio de Janeiro: FGV-RJ, v. 34, n. 3, p. 387-399, 1980.

STOUFFER, S. et al. Measurement and prediction. Princeton: Princeton University Press, 1950.

VALLE SILVA, N. Os rendimentos pessoais. In: HASENBALG, C.; VALLE SILVA, N. (Orgs.). Origens e destinos: desigualdades sociais ao longo da vida. Rio de Janeiro: Top Books. p. 431-456.

Updating the costs of not being white in Brazil. In: FONTAINE, P. M. (Org.). Race, class and power in Brazil. [S.1.]: Center for Afro-American Studies, UCLA, 1985. p. $42-55$.

O preço da cor: diferenciais raciais na distribuição de renda no Brasil. Pesquisa e Planejamento Econômico, v. 10, n. 1, p. 21-44, abr. 1980.

WORLD BANK. Living standards measurement study survey home page. Disponível em: <http://www.worldbank.org/lsms> Acesso em: 31 maio 2002. 